Suchý T., Šupová M., Klapková E., Horný L., Rýglová Š., Žaloudková M., Braun M., Sucharda Z., Ballay R., Veselý J., Chlup H., Denk F. (2Ol5) The sustainable release of vancomycin and its degradation products from nanostructured collagen/hydroxyapatit composite layers. J Pharm Sci, in press. DOI: 10.1016/SOO22-3549(15)00175-6, Publisher link: http://dx.doi.org/10.1016/S0022-3549(15)00175-6

Accepted manuscript version

\title{
The sustainable release of Vancomycin and its degradation products from nanostructured collagen/hydroxyapatite composite layers
}

\author{
Tomáš Suchý1,3, Monika Šupová1, Eva Klapková2, Lukáš Horný3, Šárka Rýglová1, Margit Žaloudková1, \\ Martin Braun ${ }^{1}$, Zbyněk Sucharda ${ }^{1}$, Rastislav Ballay ${ }^{4}$, Jan Veselý ${ }^{3}$, Hynek Chlup ${ }^{3}$, František Denk ${ }^{1}$
}

\begin{abstract}
${ }^{1}$ Department of Composites and Carbon Materials, Institute of Rock Structure and Mechanics, Academy of Sciences of the Czech Republic, v.v.i. V Holešovičkách 41, Prague 8, Czech Republic

${ }^{2}$ Department of Medical Chemistry and Clinical Biochemistry, Charles University, 2nd Medical School and University Hospital Motol, V Úvalu 84, Prague 5, Czech Republic

${ }^{3}$ Faculty of Mechanical Engineering, Czech Technical University in Prague, Technická 4, Prague 6, Czech Republic

${ }^{4} 1^{\text {st }}$ Department of Orthopaedics, $1^{\text {st }}$ Faculty of Medicine, Charles University in Prague and Motol University Hospital, V Úvalu 84, Prague 5, Czech Republic
\end{abstract}

Corresponding author: Tomáš Suchý, Department of Composites and Carbon Materials, Institute of Rock Structure and Mechanics, Academy of Sciences of the Czech Republic, v.v.i. V Holešovičkách 41, Prague 8, 182 09, Czech Republic, suchyt@irsm.cas.cz

\begin{abstract}
Infections of the musculoskeletal system present a serious problem with regard to the field of orthopaedic and trauma medicine. The aim of the experiment described in this study is to develop a resorbable nanostructured composite layer with the controlled elution of antibiotics. The layer is composed of collagen, hydroxyapatite nanoparticles and Vancomycin hydrochloride (10wt\%). The stability of the collagen was enhanced by means of cross-linking. Four cross-linking agents were studied, namely an ethanol solution, a phosphate buffer solution of $\mathrm{N}$-(3-dimethylaminopropyl)- $\mathrm{N}^{\prime}$ ethylcarbodiimide hydrochloride/N-hydroxysuccinimide, genipin and nordihydroguaiaretic acid. High performance liquid chromatography was employed so as to characterize the in vitro release rates of the Vancomycin and its crystalline degradation antibiotically inactive products over a 21-day period. The maximum concentration of the released active form of Vancomycin (approximately $265 \mathrm{mg} / \mathrm{l}$ ) exceeded the minimum inhibitory concentration (MIC) up to an order of 17 times without triggering the burst releasing effect. At the end of the experiment the MIC was exceeded by up to 6 times (approximately $100 \mathrm{mg} / \mathrm{I}$ ). It was determined that the modification of collagen with hydroxyapatite nanoparticles does not negatively influence the sustainable release of Vancomycin. The balance of Vancomycin and its degradation products was observed following 14 days of incubation.
\end{abstract}

\section{Keywords}

Antiinfectives; HPLC; Coating; Controlled release; Degradation products; Drug delivery systems; Nanoparticles; Pharmacokinetics; Polymeric drug delivery systems 
Suchý T., Šupová M., Klapková E., Horný L., Rýglová Š., Žaloudková M., Braun M., Sucharda Z., Ballay R., Veselý J., Chlup H., Denk F. (2Ol5) The sustainable release of vancomycin and its degradation products from nanostructured collagen/hydroxyapatit composite layers. J Pharm Sci, in press. DOI: 10.1016/SOO22-3549(15)00175-6, Publisher link: http://dx.doi.org/10.1016/S0022-3549(15)00175-6

Accepted manuscript version.

\section{Introduction}

The infection of implanted endoprostheses represents a serious problem as far as orthopaedic and trauma surgery is concerned. Indeed, it is often associated with devastating consequences and treatment presents a substantial challenge for surgeons. Despite the fact that a range of more or less successful methods are available, none of them is considered a method of first choice. One of the ways in which to increase the efficacy of the therapy is to employ a local antibiotic delivery system. The local antibiotic treatment of prosthetic joint infection as opposed to the use of systemic antibiotics enjoys the advantage of achieving high antibiotic concentrations which exceed the minimum inhibitory concentration (MIC) without increasing the level of systemic toxicity. Local carriers of antibiotics used in the field of orthopaedics are classified according to composition as synthetic and natural polymers, ceramics, composites and bone grafts. Since the 1950s Vancomycin (glycopeptide antibiotics) has been used to treat severe infections caused by gram-positive micro-organisms. As a result of the increasing incidence of methicillin/oxacillin-resistant Staphylococcus aureus (MRSA) in chronically and seriously ill patients, the use of Vancomycin has increased significantly. Vancomycin-resistant Staphylococcus aureus (VRSA) is most common in elderly patients suffering from leg ulcers or pressure sores, especially those patients with a history of Vancomycin-resistant enterococci. One possible explanation as to why infections caused by VRSA are becoming more commonly reported is that Vancomycin breaks down over time to form crystalline degradation products (CDP-1s - see Fig. 1.) which are devoid of antibacterial activity due to the disruption of the hydrogen bond at the site of the antigen binding site of a specific Vancomycin receptor [1]; at the same time the efficacy of the active form of Vancomycin decreases. During in vitro exposure at a temperature of $20-25^{\circ} \mathrm{C}$, up to $50 \%$ of Vancomycin is converted to CDP-1s within 16 hours and $90 \%$ of Vancomycin is converted to CDP-1s within 40 hours. In addition, an acidic $\mathrm{pH}$ of 4.1 to 4.2 contributes towards the formation of CDP-1s, two CDP-1 isomers of which exist: CDP-1M (major) and CDP-1m (minor) - conformational isomers - formed by the hydrolytic loss of ammonia. CDPs are structurally similar to Vancomycin, i.e. they exhibit two carboxyl groups. The accumulation of CDP-1s can lead to toxic tissue damage and may also result in the failure of therapy due to the occurrence of sub-therapeutic levels of Vancomycin.
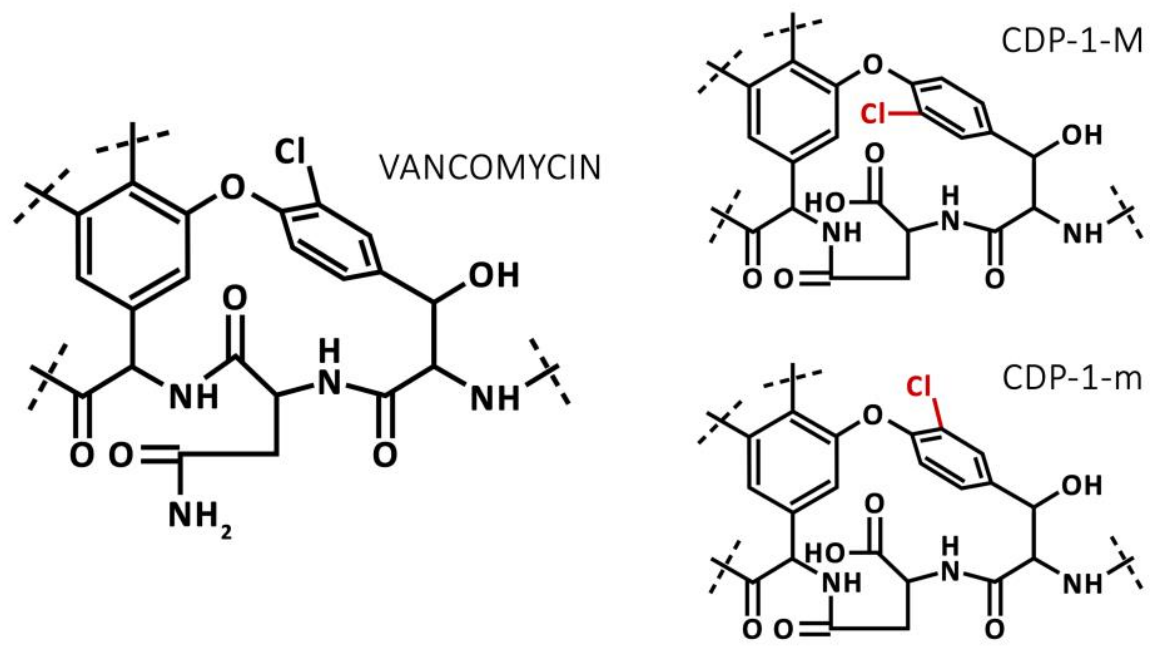

Fig. 1 Vancomycin structures and its degradation products. 
Suchý T., Šupová M., Klapková E., Horný L., Rýglová Š., Žaloudková M., Braun M., Sucharda Z., Ballay R., Veselý J., Chlup H., Denk F. (2Ol5) The sustainable release of vancomycin and its degradation products from nanostructured collagen/hydroxyapatit composite layers. J Pharm Sci, in press. DOI: 10.1016/SOO22-3549(15)00175-6, Publisher link: http://dx.doi.org/10.1016/SO022-3549(15)00175-6

Accepted manuscript version.

Polymethylmethacrylate (PMMA) bone cement is commonly used for the fixation of joint prostheses. In the late 1960 s antibiotic impregnated cement was considered to provide prevention from infection [2]. However, it has since been established that PMMA provides an initial burst release of antibiotics with the larger part of the loaded antibiotic remaining within the cement thus preventing PMMA from providing an effective long-term anti-inflammatory function $[3,4]$. A further problem consists of the exothermic reaction of the bone cement during its polymerization which, to a significant extent, limits the types of antibiotics that can be effectively incorporated into the cement [5]. Moreover, it may accelerate the conversion of Vancomycin into crystalline degradation products and thus lead to a significant decrease in antimicrobial activity. In addition, a number of investigators have demonstrated that biofilm can be easily formed on the surface of antibiotic-loaded cement [6-8]. Thus, with a view to overcoming these disadvantages, the study focuses on the development of a re-sorbable nanostructured composite layer based on natural and synthetic polymers [4, 9] modified by means of calcium phosphate nanoparticles [10].

The aim of the study was to develop a biodegradable nanostructured composite layer with the controlled elution of Vancomycin. It is expected that such a layer will be used particularly in the case of known prosthetic joint infections or as a preventative procedure regarding primary joint replacement in a potentially infected site. The layer will provide a bone/implant (titanium alloy) bioactive interface which will enhance the physiological healing process, will be capable of filling bone defects and will act as a powerful antibacterial agent against those microorganisms susceptible to Vancomycin. The layer is composed of collagen (type I, isolated from calf skin), hydroxyapatite nanoparticles and Vancomycin hydrochloride. The material composition of the various layers imitates the composition of real bone tissue. Three different layer compositions were analysed the aim of which was to verify the potential effect of hydroxyapatite on the release of Vancomycin. However, it is important to mention at this point that the application of collagen in the field of tissue engineering is limited due to its poor mechanical properties, high rate of swelling in water, low structural stability and low resistivity against the enzymatic degradation of its untreated form [11]. A number of crosslinking agents can be used in order to both improve its mechanical properties and slow down the biodegradation rate of collagen-based materials. Chemical cross-linking is achieved principally through covalent amine/imine linkage. The cross-linking agents and conditions employed in this study include $\mathrm{N}$-(3-dimethylaminopropyl)-N-ethylcarbodiimide hydrochloride (EDC)/N-hydroxysuccinimide (NHS) [12], genipin (GEN) [13] and nordihydroguaiaretic acid (NDGA) [14,15], all of which are commonly used cross-linking agents. The structural properties, swelling ratio and degradation rate of these scaffolds were investigated in detail and subsequently compared in order to determine the optimal cross-linking conditions. The study aimed principally to verify whether the local concentration of the Vancomycin released exceeded the MIC for Vancomycin-resistant Staphylococcus aureus (VRSA >16mg/l) and to monitor the concentrations of active forms of Vancomycin and its degradation products released from the nanostructured composite layer used as local antibiotic carriers in the treatment of osteomyelitis. The objective evaluation of antibiotically active and antibiotically inactive forms of Vancomycin is a 
Suchý T., Šupová M., Klapková E., Horný L., Rýglová Š., Žaloudková M., Braun M., Sucharda Z., Ballay R., Veselý J., Chlup H., Denk F. (2Ol5) The sustainable release of vancomycin and its degradation products from nanostructured collagen/hydroxyapatit composite layers. J Pharm Sci, in press. DOI: 10.1016/SOO22-3549(15)00175-6, Publisher link: http://dx.doi.org/10.1016/SO022-3549(15)00175-6

Accepted manuscript version.

very important issue. Moreover, crystalline degradation products cross-react with certain immunoassays that use polyclonal antibodies thus resulting in falsely elevated results [16]. High performance liquid chromatography (HPLC) provides an effective tool for the quantitative and qualitative analysis of Vancomycin and CDP-1s. At the end of the 1990s Backes et al. [16] developed an HPLC method to quantitate Vancomycin and CDP-1s in serum of renal patients. However, their method has not been adapted to studies which deal with the evaluation of local Vancomycine carriers. The monitoring of the antibiotically active form of Vancomycin and its inactive degradation products is unfortunately often ignored (e.g. [17-21]).

\section{Materials and methods}

\subsection{Fabrication of collagen layers and cross-linking agent selection}

A collagen (type I, calf skin, VUP Medical, Czech Republic) solution (5wt\%) was prepared by the swelling of collagen in a phosphate buffer saline solution (PBS, $0.0027 \mathrm{M}$ potassium chloride and $0.137 \mathrm{M}$ sodium chloride, pH 7.4 at $25^{\circ} \mathrm{C}$ ) (Sigma Aldrich, Germany) and ethanol (Penta, Czech Republic) (1/1, v/v) and homogenized by means of a disintegrator (10000rpm, $10 \mathrm{~min}$ ). The dispersion was transferred to silicone moulds with inner dimensions of $30 \times 70 \times 4 \mathrm{~mm}$ and dried in a laminar box until a constant sample weight was achieved (up to 48 hours at room temperature). The collagen layers were subsequently cross-linked employing four different chemical treatments. The first group of samples (EDC/NHS/ETOH) was cross-linked with an ethanol solution containing EDC and NHS at a weight ratio of $4: 1$ (EDC: $4.08 \mathrm{mg} / \mathrm{ml}$ and NHS: $1.02 \mathrm{mg} / \mathrm{ml}$ ); the EDC and NHS (Sigma Aldrich, Germany) were used as received. The second group (EDC/NHS/PBS) was cross-linked using PBS containing EDC/NHS (weight ratio $4: 1$, EDC: $4.08 \mathrm{mg} / \mathrm{ml}$ and NHS: $1.02 \mathrm{mg} / \mathrm{ml}$ ). The third group was cross-linked using PBS containing genipin (Sigma Aldrich, Germany) at a concentration of $1.34 \mathrm{mg} / \mathrm{ml}$. The last group was cross-linked by means of PBS containing nordihydroguaiaretic acid (Cayman Chemical, USA) at a concentration of $5 \mathrm{mg} / \mathrm{ml}$ (5mg NDGA, $0.9 \mathrm{ml}$ PBS, $0.1 \mathrm{ml} 0.1 \mathrm{~N} \mathrm{NaOH}$ ). Following a reaction period of 24 hours at room temperature (using an orbital shaker, 180rpm), all the layers were washed in $0.1 \mathrm{M} \mathrm{Na} \mathrm{NaO}_{4}(2 \times 45$ $\mathrm{min}$ ), followed by rinsing using deionised water (30 $\mathrm{min}$ ), placed in the laminar box and dried at room temperature until a constant weight was achieved (up to 48 hours).

\subsection{Modification of the collagen layers by means of hydroxyapatite and Vancomycin}

Following the selection of suitable cross-linking conditions, collagen layers modified by means of hydroxyapatite (HA) and Vancomycin were prepared. HA nanoparticles (average size 150nm, SigmaAldrich) in the amount of $0 \mathrm{wt} \%(0 \% \mathrm{HA}), 5 \mathrm{wt} \%(5 \% \mathrm{HA})$ and $15 \mathrm{wt} \%(15 \% \mathrm{HA})$ and $10 \mathrm{wt} \%$ of Vancomycin hydrochloride (Vancomycin Mylan ${ }^{\circledR}$, Mylan S.A.S, France) were used for the preparation of the layer intended for use as a nano-structured interface covering the surface of functional implants with enhanced osteo-integration and a prophylactic effect. A collagen solution (5wt\%) was prepared by the swelling of collagen in a PBS/ethanol solution $(1 / 1, v / v)$ and homogenization was achieved using a 
Suchý T., Šupová M., Klapková E., Horný L., Rýglová Š., Žaloudková M., Braun M., Sucharda Z., Ballay R., Veselý J., Chlup H., Denk F. (2Ol5) The sustainable release of vancomycin and its degradation products from nanostructured collagen/hydroxyapatit composite layers. J Pharm Sci, in press. DOI: 10.1016/SOO22-3549(15)00175-6, Publisher link: http://dx.doi.org/10.1016/SO022-3549(15)00175-6

Accepted manuscript version.

disintegrator (10 000rpm, $10 \mathrm{~min})$. HA in the amount of 0,5 and $15 \mathrm{wt} \%$ was then added to this solution. Finally, Vancomycin was added to the solution in the amount of $10 \%$ wt of the final weight of collagen (in the case of $0 \% \mathrm{HA}$ ) or the final weight of collagen with $\mathrm{HA}$ (in the case of $5 \% \mathrm{HA}$ and $15 \% \mathrm{HA}$ ). The final solution was homogenized using the disintegrator (10 000rpm, $10 \mathrm{~min}$ ); each solution was packed into polyethylene containers and deposited in a freezer for 24 hours at a temperature of $-15^{\circ} \mathrm{C}$ and finally lyophilized (BenchTop 4KZL, VirTis). The weight of each separate sample was approximately 4g. The condenser of the freeze-drying device was initially precooled $\left(-60^{\circ} \mathrm{C}\right)$ then evacuated to a pressure of close to $\mathrm{OPa}$ and finally cooled to a temperature of $-105^{\circ} \mathrm{C}$. Following this procedure the samples were placed in glass containers. The containers were then evacuated within 10 seconds. The lyophylization process $\left(-105^{\circ} \mathrm{C}, 1.3 \mathrm{~Pa}\right)$ took a total of 48 hours. The final temperature of the glass containers following the process was in balance with the temperature of the laboratory. The stability of the lyophilized samples was enhanced by a suitable cross-linking procedure, i.e. in this case EDC/NHS/PBS (see previous paragraph). The residual water content of the samples following crosslinking was approx. $5 \mathrm{wt}$. \% (determined by the weighing of the samples dried at $105^{\circ} \mathrm{C}$ for 4 hours). No statistically significant differences were detected between the residual water content of the individual samples $(p<0.05)$.

\subsection{Mechanical testing}

In order to determine the optimal cross-linking method, mechanical properties were evaluated by means of the conducting of uniaxial tensile tests of rectangular strips of the layers (the average width of the samples was approximately $10-50 \mathrm{~mm}$ ). During the test procedure the value of strain at failure (the maximum strain sustained by the material before breaking where strain is defined as the ratio of the elongation of the sample to reference length), the ultimate tensile strength (the maximum nominal stress sustained by the material; nominal stress is defined as the ratio of applied force to the reference cross-section of a sample) and the modulus of elasticity (the slope of the tangent made to a stressstrain relationship on the initial linear part) were determined. Tensile tests were conducted using a Zwick/Roell multipurpose testing machine equipped with a built-in video extensometer. By using contrasting marks on the surface of samples, the video extensometer automatically determined the reference length and elongation of the samples. Tensile experiments were conducted at a constant clamp velocity of $0.1 \mathrm{~mm} / \mathrm{s}$. The loading force was measured by a U9B ( $\pm 250 \mathrm{~N}, \mathrm{HBM}$, Germany) force transducer.

\subsection{Structural stability}

The initial analysis of collagen layer stability was conducted by means of degradation testing. The samples were immersed in blood plasma ( 8 donors of different blood group, sex and age) and incubated at $37^{\circ} \mathrm{C}$ and a $5 \% \mathrm{CO}_{2}$ atmosphere ( $\mathrm{DH} \mathrm{CO}_{2}$ incubator, Thermo Scientific) with antibiotics (penicillin/streptomycin) for 6 hours and for 1, 3, 10, 15 and 30 days. The volume of the medium was maintained at the same weight/volume ratio of $30 \mathrm{mg} / 15 \mathrm{ml}$. The extent of in vitro degradation was calculated according to the following equation: $D=\frac{W_{0}-W_{t}}{W_{0}} 100[\%]$, where $D$ is the degradation rate, 
Suchý T., Šupová M., Klapková E., Horný L., Rýglová Š., Žaloudková M., Braun M., Sucharda Z., Ballay R., Veselý J., Chlup H., Denk F. (2Ol5) The sustainable release of vancomycin and its degradation products from nanostructured collagen/hydroxyapatit composite layers. J Pharm Sci, in press. DOI: 10.1016/SOO22-3549(15)00175-6, Publisher link: http://dx.doi.org/10.1016/SO022-3549(15)00175-6

Accepted manuscript version.

$W_{o}$ is the initial dried weight of the sample and $W_{t}$ is the dried weight of the sample after degradation $(n=3)$. The swelling ratio $\left(E_{s w}\right)$ was calculated using the following equation: $E_{s w}=\frac{W_{s w}-W_{0}}{W_{0}} 100[\%]$, where $W_{o}$ is the initial dried weight of the sample and $W_{s w}$ is the weight of the swollen sample $(n=3)$. The weight of the swollen samples was measured following the removal of each sample from the medium and following a 1 minute delay and the removal of any excessive medium surrounding the sample; the dried weight of the samples was measured following lyophilisation.

Degradation tests under physiological conditions were used as the second way in which to assess the stability of the collagenous layers, and UV-VIS spectrophotometry was employed for the quantification of the free amino groups released during the degradation of the samples immersed in the PBS $\left(37^{\circ} \mathrm{C}\right.$, pH 7.4) and 2,4,6-trinitrobenzenesulphonic acid (TNBS, Sigma Aldrich) solution. PBS was collected after 2, 6 and 24 hours and after 4, 10, 15 and 30 days. The evaluation of the samples was conducted using calibration curve methodology in which the L-lysine (Sigma Aldrich) solution was used as the standard solution.

\subsection{Vancomycin release}

An investigation was conducted of the in vitro release of Vancomycin from EDC/NHS in PBS cross-linked layers. Six samples of each type of Vancomycin-impregnated samples were placed on a sterile gauze pad and were subsequently firmly caulked and transferred to separate test tubes with a weight/volume ratio of $0.17 \mathrm{~g} / 20 \mathrm{ml}$ of PBS ( $\mathrm{pH} 7.4$ ) and finally placed in an incubator at a temperature of $37^{\circ} \mathrm{C}$. The solid phase extraction method and high-performance liquid chromatographic analysis (HPLC on an Agilent 1200 series system equipped with a diode array detector - DAD, Agilent Technologies, USA) were employed in order to characterize the in vitro release rates of the Vancomycin and its crystalline degradation antibiotically inactive products over a 21-day period. The HPLC method is described in an article written by Melichercik et al. [22].

\subsection{Statistical evaluation}

Statistically significant differences were investigated principally by means of non-parametric methods (STATGRAPHICS Centurion XV, StatPoint, USA) due to the problematical nature of the verification of the normality of the assessed data (Chi-squared and Shapiro-Wilk $W$ tests) or the violation of homoscedasticity (Bartlett's, Cochran's and Leven's tests); the Kruskal-Wallis test was employed for this purpose and the Mann-Whitney W test was used for the conducting of post hoc analysis. In the case of normally distributed data sets and a successful variance check, statistically significant differences were investigated using Fisher's least significant difference (LSD) and Duncan's multiple comparison procedures. All the variance analysis was performed at a $95 \%$ confidence level ( $p$ values less than 0.05 were considered to be significant. Mean values were calculated as medians for the most part and the interquartile range (IQR) was used as the measure of statistical variance. For reasons of ease of comparison, mean values were also calculated as arithmetical averages in some cases. In the case of Box-and-Whiskers plots, the boxes indicate the interquartile range and the whiskers the 10-90 
Suchý T., Šupová M., Klapková E., Horný L., Rýglová Š., Žaloudková M., Braun M., Sucharda Z., Ballay R., Veselý J., Chlup H., Denk F. (2Ol5) The sustainable release of vancomycin and its degradation products from nanostructured collagen/hydroxyapatit composite layers. J Pharm Sci, in press. DOI: 10.1016/SOO22-3549(15)00175-6, Publisher link: http://dx.doi.org/10.1016/SO022-3549(15)00175-6

Accepted manuscript version.

percentile range. The outside dots indicate values more than 1.5 times the IQR above or below the boxes, and any point more than 3 times the IQR above or below a box is indicated by a point symbol with superimposed plus signs; a vertical line is drawn at the median point. A plus sign inside a box indicates the arithmetical average.

\section{Results and discussion}

\subsection{Mechanical testing}

Each tested group of differently cross-linked samples (EDC/NHS/ETOH, EDC/NHS/PBS, GEN, NDGA) and original non cross-linked samples (NON) consisted of 8 samples. The stress-strain relationships obtained through uniaxial tensile testing are depicted in Fig. 2. No statistical differences $(p=0.05)$ were determined between groups for strain at a failure (Fig. 3). With the exception of the EDC/NHS/PBS variant, all the cross-linked samples exhibited a statistically significant increase in ultimate tensile strength (Fig. 3). A similar result was observed in the case of the modulus of elasticity. Here again the determined modulus of elasticity values do not prove any significant effect of EDC/NHS/PBS crosslinking on macroscopic mechanical properties. In contrast to the increase in tensile strength and modulus of those samples cross-linked using EDC/NHS/ETOH, genipin and NDGA, there was no observation of the expected effect of cross-linking using EDC/NHS/PBS. The observed decrease in tensile strength and modulus in comparison with that of the other cross-linked samples could be explained by the formation of local stress concentrations due to the early failure of brittle parts of the collagen layers formed following extensive cross-linking subsequent to the application of a higher concentration of EDC/NHS in the aqueous environment [23, 24]. The effect of different cross-linking agents was further analysed by means of the methods described below.

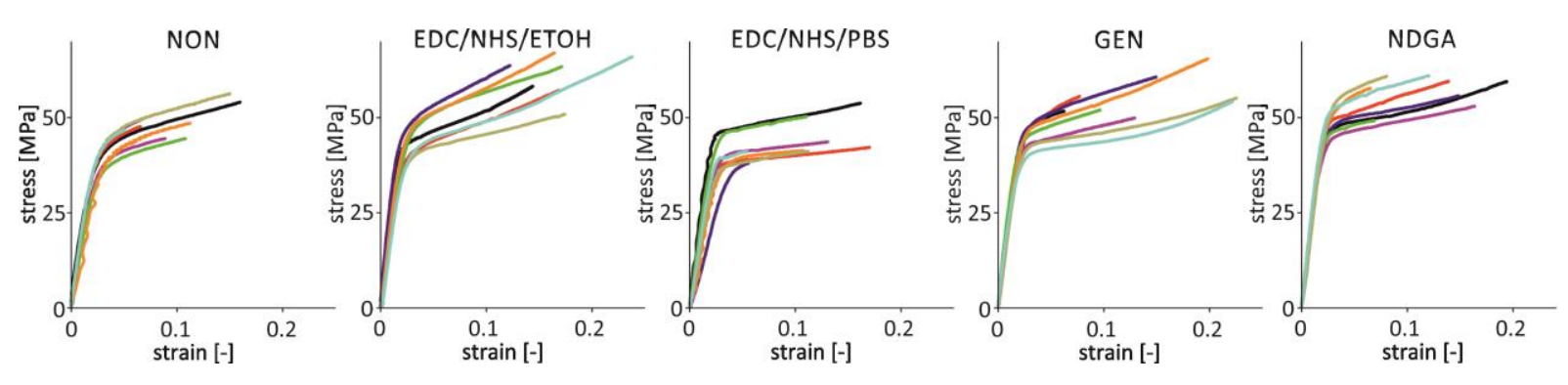

Fig. 2 Stress-strain relationships obtained via uniaxial tensile testing. 
Suchý T., Šupová M., Klapková E., Horný L., Rýglová Š., Žaloudková M., Braun M., Sucharda Z., Ballay R., Veselý J., Chlup H., Denk F. (2Ol5) The sustainable release of vancomycin and its degradation products from nanostructured collagen/hydroxyapatit composite layers. J Pharm Sci, in press. DOI: 10.1016/SOO22-3549(15)00175-6, Publisher link: http://dx.doi.org/10.1016/S0022-3549(15)00175-6

Accepted manuscript version.
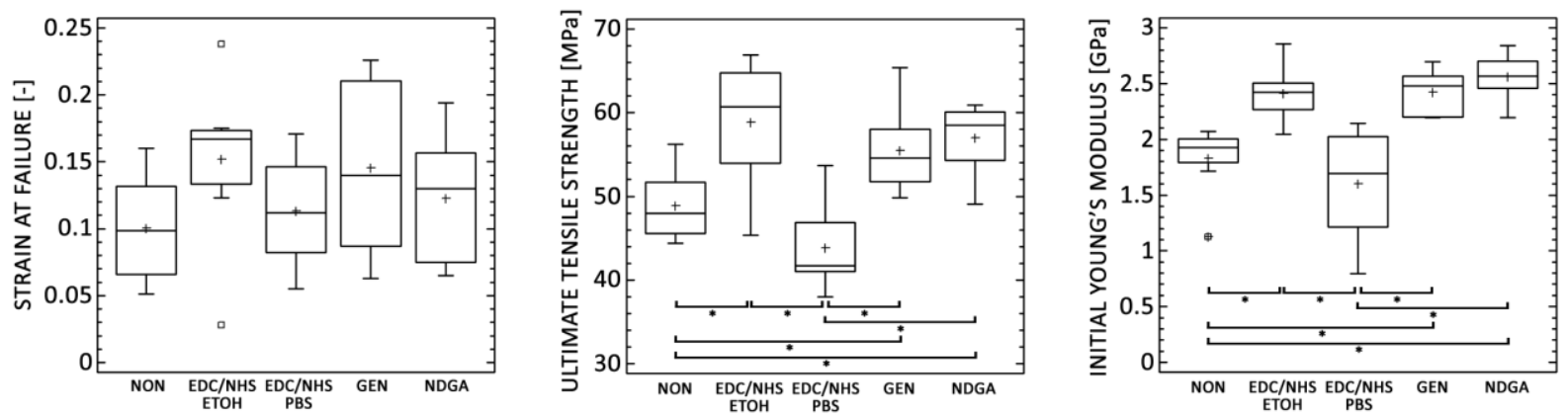

Fig. 3 Mechanical properties of collagen layers. Box-plots comparing strain at a failure, ultimate tensile strength and initial modulus of elasticity. The sign "*” denotes statistically significant differences (ultimate tensile strength: LSD and Duncan, 0.05; initial Young's modulus: Mann-Whitney, 0.05).

\subsection{Structural stability}

Accelerated mass loss (Fig. 4) was observed in the non-cross-linked samples (approximately 90\%) as well as in the EDC/NHS/ETOH cross-linked samples (approximately 80\%). The degradation rate of these samples increased markedly as soon as on the first day (after 6 hours a mass loss of up to $20 \%$ in the case of the non-cross-linked samples) with a further increase in the next 1 to 10 days (to a further $40 \%)$. In contrast, in the case of cross-linking with EDC/NHS/PBS, NDGA and GEN the degradation rate did not change significantly from 1 to 30 days. The accelerated mass loss in the case of the $\mathrm{EDC} / \mathrm{NHS} / \mathrm{ETOH}$ cross-linked samples may indicate an insufficient rate of collagen cross-linking.

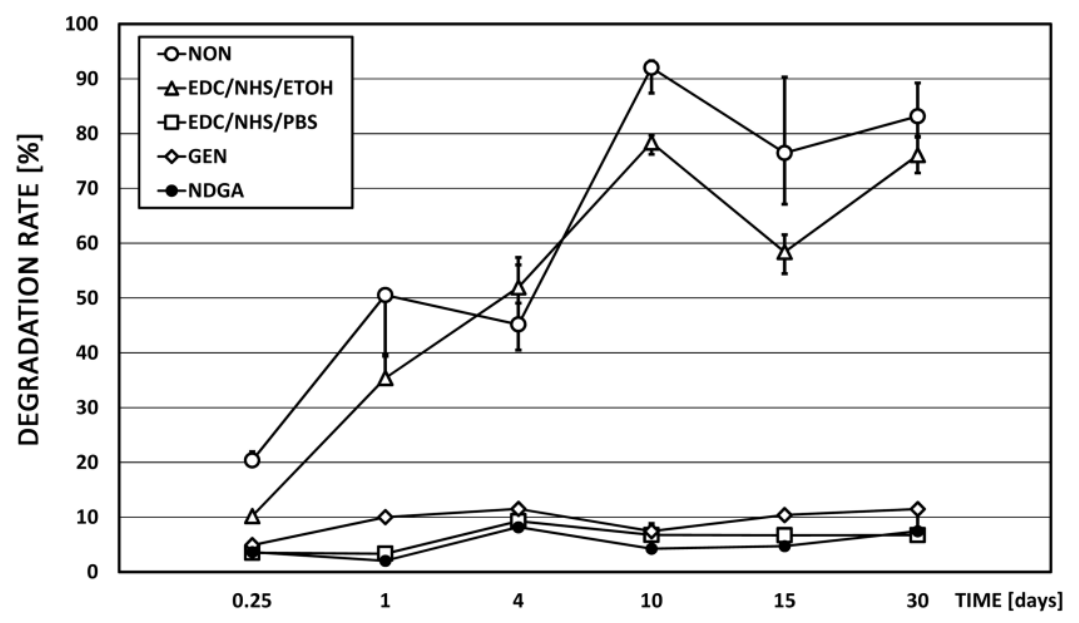

Fig. 4 Degradation rates (median, IQR) of non-cross-linked and cross-linked collagen samples. With the exception of EDC/NHS/ETOH, the degradation rate of all the cross-linked samples was significantly lower than that of the non-cross-linked samples (Mann-Whitney, 0.05).

The non-cross-linked layers and those layers cross-linked with EDC/NHS/ETOH exhibited the highest swelling ratios, i.e. $540 \%$ and $750 \%$ respectively, as soon as after 6 hours (Fig. 5). These samples demonstrated a decreasing swelling ratio tendency over time. This might have been caused by extensive material degradation (less material available for swelling). In contrast, the EDC/NHS/PBS, GEN and NDGA samples exhibited the lowest statistically significant differences $(p=0.05)$. Indeed, these three groups demonstrated very similar behaviour - all the samples absorbed approximately triple 
Suchý T., Šupová M., Klapková E., Horný L., Rýglová Š., Žaloudková M., Braun M., Sucharda Z., Ballay R., Veselý J., Chlup H., Denk F. (2Ol5) The sustainable release of vancomycin and its degradation products from nanostructured collagen/hydroxyapatit composite layers. J Pharm Sci, in press. DOI: 10.1016/SOO22-3549(15)00175-6, Publisher link: http://dx.doi.org/10.1016/S0022-3549(15)00175-6

Accepted manuscript version.

their weight within the first 6 hours of the experiment and, subsequently, the swelling ratio stabilised. This behaviour would seem to demonstrate the enhancement of structural stability following the application of the cross-linking procedure.

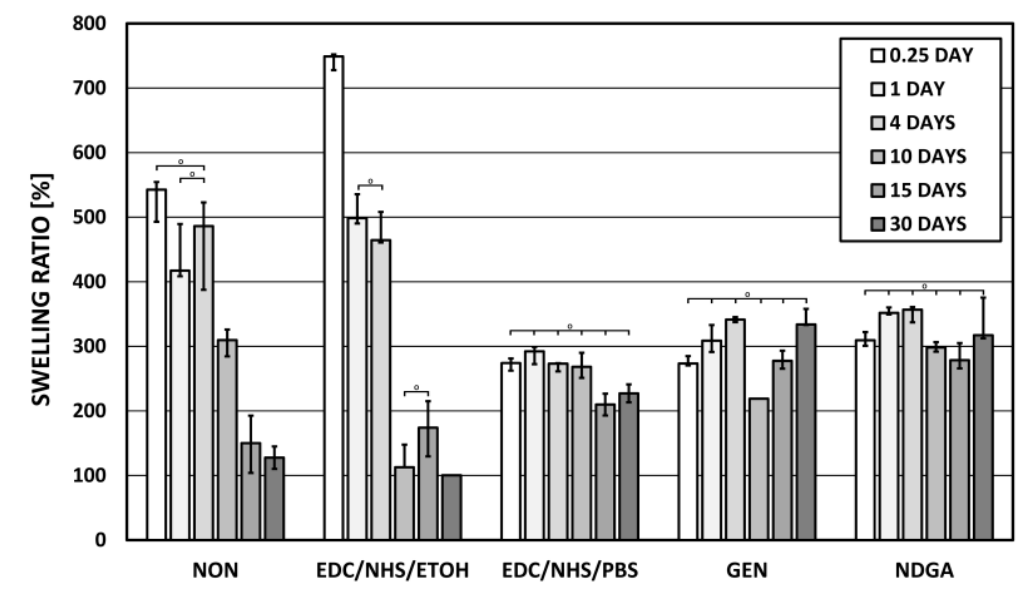

Fig. 5 The swelling ratios of non-crosslinked and cross-linked collagen samples (median, IQR). The sign "o" denotes data sets with no statistically significant differences (NON and EDC/NHS/ETOH: Mann-Whitney, 0.05; EDC/NHS/PBS, GEN and NDGA: Kruskal-Wallis, 0.05).

Based on the relative comparison of released amino acids (Fig. 6) the materials studied can be divided into three different groups. Non-cross-linked samples and the EDC/NHS/ETOH samples $(p=0.05)$ make up a group featuring the highest concentration of released amino acids (i.e. the lowest level of resistance to decomposition under physiological conditions). This corresponds with the results of the blood plasma experiment. The second group, i.e. those samples cross-linked with NDGA $(p=0.05)$ exhibited the highest rates of cross-linking. The third "average" group was made up of those samples cross-linked by means of the EDC/NHS/PBS and genipin cross-linked samples.

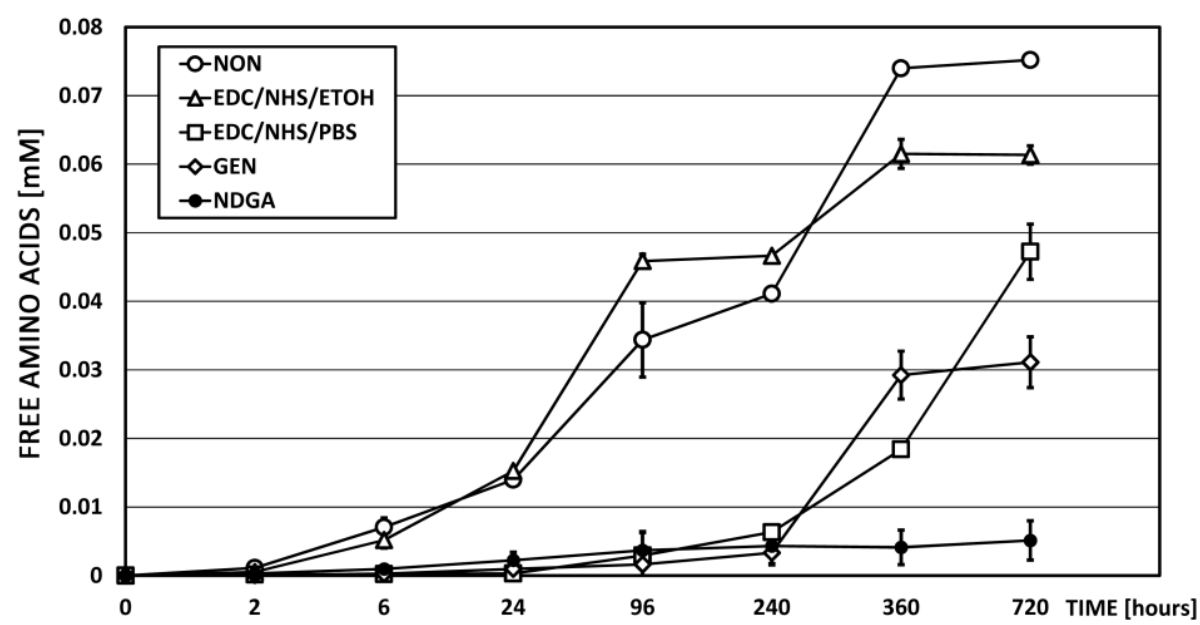

Fig. 6 The concentration of amino acids released from non-cross-linked and cross-linked samples (arithmetical average, standard deviation).

The degradation rate, swelling ratio and the determination of released amino acids provide important indices in terms of the evaluation of the structural stability of the collagen layers. For all three analyses, the EDC/NHS/PBS solution was chosen as the optimal cross-linking agent for the further preparation of collagen layers modified by means of Vancomycin and hydroxyapatite nanoparticles. The group of 
Suchý T., Šupová M., Klapková E., Horný L., Rýglová Š., Žaloudková M., Braun M., Sucharda Z., Ballay R., Veselý J., Chlup H., Denk F. (2Ol5) The sustainable release of vancomycin and its degradation products from nanostructured collagen/hydroxyapatit composite layers. J Pharm Sci, in press. DOI: 10.1016/SOO22-3549(15)00175-6, Publisher link: http://dx.doi.org/10.1016/SO022-3549(15)00175-6

Accepted manuscript version.

three cross-linking agents (EDC/NHS/PBS, NDGA and GEN) evinced very similar properties when subjected to the evaluation of mechanical properties, degradation rate, swelling ratio and concentration of released free amino acids. However, the results of the in vitro testing of NDGA crosslinked materials, published in literature $[25,26]$ are ambiguous and often antagonistic. Importantly, in addition to the successful cross-linking effect of genipin coupled with its low toxicity, the related economic issues must be carefully considered.

\subsection{Vancomycin release}

Following the assessment of optimal cross-linking conditions, the preparation procedure was applied to samples with Vancomycin. The kinetics of Vancomycin release can be optimized by means of changing the inner structure of the layers through changing the lyophylization parameters. Porosity and the related specific surface area make up one of the key parameters affecting the elution of antibiotics. Antibiotic release may also be controlled via the optimization of collagen stability by means of changing the parameters of the cross-linking process. A wide range of other ways exist with regard to controlling the release of Vancomycin. The approach applied in this study was to employ the same preparation procedure for all the samples, i.e. to prepare samples with the same structural properties but differing in terms of the amount of hydroxyapatite. Initial measurements, three hours following the application of the samples, revealed average concentrations of up to $18 \mathrm{mg} / \mathrm{l}$ for all the samples (Fig. 7). The highest average concentration of Vancomycin (approximately $250 \mathrm{mg} / \mathrm{l}$ ) was achieved after 8 days while levels of CDP-1M were determined to be up to $120 \mathrm{mg} / \mathrm{I}$ and those of CDP- $1 \mathrm{~m}$ up to $18 \mathrm{mg} / \mathrm{l}$. After 14 days of incubation the average concentration of CDP-1M was found to be similar to that of Vancomycin $(\sim 180 \mathrm{mg} / \mathrm{l})$. Interestingly, Vancomycin was converted into its degradation products at a much slower rate than reported by [22] who observed balance as soon as after just 6 days. Subsequently, the concentration of Vancomycin began to decrease and CDP-1M levels increase up to the end of the measurement period on day 21. From the $4^{\text {th }}$ day following the placement of the carriers into tubes, a gradual and disproportionate increase in CDP-1s was observed in the surrounding buffer which might have been due to the conversion of the released Vancomycin into CDP-1s. Consequently, Vancomycin was evident in much lower concentrations at the end of the experiment. However, despite the considerable tendency of Vancomycin towards crystalline thermal degradation, levels of the released active form of Vancomycin remained above the MIC for VRSA for more than 3 weeks. The maximum concentration of the released active form of Vancomycin (approximately $265 \mathrm{mg} / \mathrm{l}$ ) exceeded the MIC by up to 17 times. At the end of the experiment ( 21 days) the MIC was exceeded by up to 6 times (approximately $100 \mathrm{mg} / \mathrm{l}$ ). The highest concentration level was obtained following a period of 192 hours and without the burst releasing effect. These results would seem to suggest that the local application of high-dose Vancomycin via the use of drug delivery carriers is a safe therapeutic method for the treatment of osteomyelitis that prevents the development of bacterial resistance. 
Suchý T., Šupová M., Klapková E., Horný L., Rýglová Š., Žaloudková M., Braun M., Sucharda Z., Ballay R., Veselý J., Chlup H., Denk F. (2Ol5) The sustainable release of vancomycin and its degradation products from nanostructured collagen/hydroxyapatit composite layers. J Pharm Sci, in press. DOI: 10.1016/SOO22-3549(15)00175-6, Publisher link: http://dx.doi.org/10.1016/S0022-3549(15)00175-6

Accepted manuscript version.
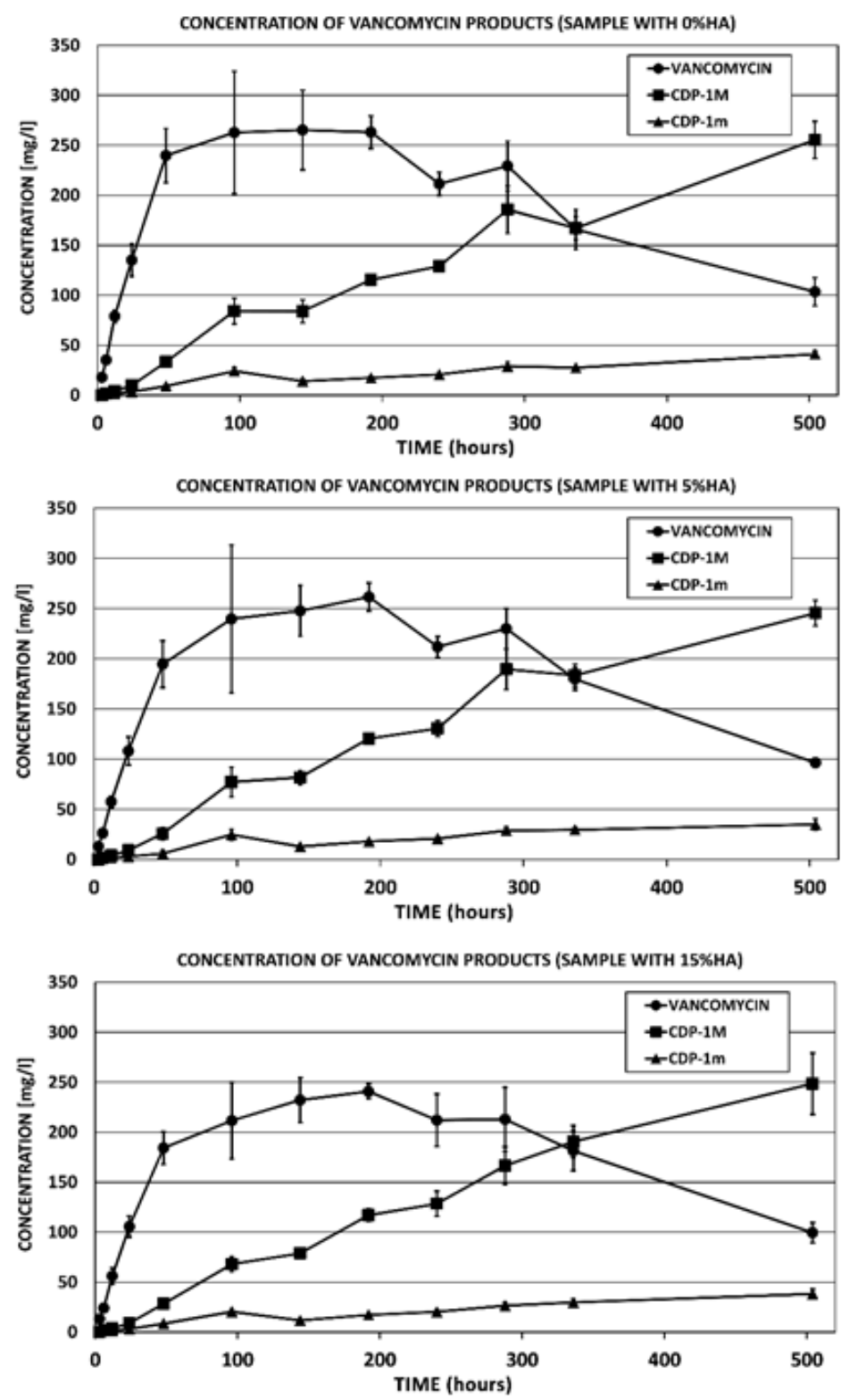

Fig. 7 The concentration of released Vancomycin, CDP-1M and CDP-1m degradation products (arithmetical average, standard deviation).

The addition of 5 and $15 \mathrm{wt} \%$ of HA nanoparticles was found to partially influence the release of Vancomycin (Fig. 8). There is a deceleration in the rate of release during the first 48 hours. After two days no statistically significant differences were discovered between samples with and without HA. Thus, the determined levels of Vancomycin release suggest that the modification of collagen by means of HA nanoparticles does not have a strong effect on the evolution of Vancomycin. The incorporation of Vancomycin with calcium phosphate could be limited due to molecule size and, consequently, by chemical steric hindrance as mentioned by Stigter et al. [17]. In addition to the minor effect of HA nanoparticles on Vancomycin release, the modification of collagen by means of calcium phosphate particles may have a positive effect on bioactivity. In other words, the addition of HA increases the rate of osseointegration and does not negatively alter the sustainable release of Vancomycin. 
Suchý T., Šupová M., Klapková E., Horný L., Rýglová Š., Žaloudková M., Braun M., Sucharda Z., Ballay R., Veselý J., Chlup H., Denk F. (2Ol5) The sustainable release of vancomycin and its degradation products from nanostructured collagen/hydroxyapatit composite layers. J Pharm Sci, in press. DOI: 10.1016/SOO22-3549(15)00175-6, Publisher link: http://dx.doi.org/10.1016/SO022-3549(15)00175-6

Accepted manuscript version.

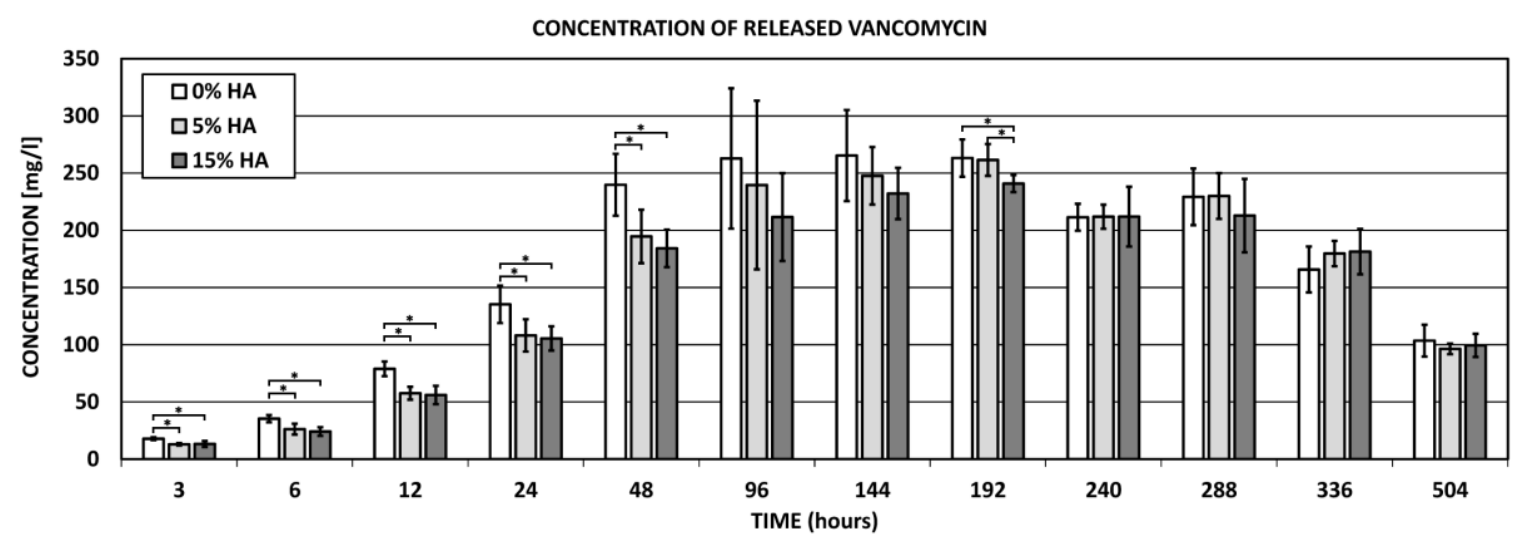

Fig. 8 The concentration of released Vancomycin from samples with 0,5 and $15 w t \%$ of hydroxyapatite (arithmetical average, standard deviation). The sign “*” denotes statistically significant differences (MannWhitney, 0.05).

The amount of the released Vancomycin and CDP-1s (determined on the basis of weight balance at the end of the experiment) was approximately $45 \mathrm{wt} \%$ for the $0 \% \mathrm{HA}, 5 \% \mathrm{HA}$ and $15 \% \mathrm{HA}$ samples. The total amount of released Vancomycin after 21 days was composed of $25 \%$ of the active form and $75 \%$ of inactive degradation products (CDP-1M: 64\%, CDP-1m: 11\%).

\section{Conclusions}

Based on the analysis of degradation rate, swelling ratio and concentration of released free amino acids, the EDC/NHS/PBS solution was chosen as the optimal cross-linking agent. In addition to the successful cross-linking effect of genipin and its low toxicity, economic considerations were also carefully considered.

Despite the considerable tendency of Vancomycin towards crystalline thermal degradation, levels of the released active form of Vancomycin remained above the MIC for VRSA for more than 3 weeks without the burst releasing effect. The maximum concentration of the released active form of Vancomycin (approx. 265mg/l) exceeded the MIC by up to 17 times. At the end of the experiment (21 days) the MIC was exceeded by up to 6 times (approx. 100 mg/l). The balance of Vancomycin and its degradation products was observed after 14 days of incubation and the conversion of Vancomycin to its degradation products was observed at a much slower rate than was reported in previous literature. The amount of released Vancomycin and its degradation products was approximately $45 \mathrm{wt} \%$ for all the sample modifications. The total amount of released Vancomycin after 21 days was composed of $25 \%$ of Vancomycin in the active form and $75 \%$ of inactive degradation products. It can therefore be concluded that the modification of collagen by means of HA nanoparticles can increase the rate of osseointegration and does not negatively influence the sustainable release of Vancomycin.

These results suggest that the local application of high-dose Vancomycin via the use of drug delivery carriers presents a safe therapeutic osteomyelitis treatment method that prevents the development 
Suchý T., Šupová M., Klapková E., Horný L., Rýglová Š., Žaloudková M., Braun M., Sucharda Z., Ballay R., Veselý J., Chlup H., Denk F. (2Ol5) The sustainable release of vancomycin and its degradation products from nanostructured collagen/hydroxyapatit composite layers. J Pharm Sci, in press. DOI: 10.1016/SOO22-3549(15)00175-6, Publisher link: http://dx.doi.org/10.1016/S0022-3549(15)00175-6

Accepted manuscript version.

of bacterial resistance. It is planned that the effect of the antibiotic concentration obtained on systemic toxicity and antimicrobial activity working against both gram-positive and gram-negative bacteria will be further evaluated and verified in future research.

\section{Acknowledgements}

This study was supported by a grant provided by the Technology Agency of the Czech Republic under project no. TA04010330. In addition, the research team gratefully acknowledges the financial support provided for our work by the long-term conceptual development of research organisations' project no. RVO: 67985891. Special thanks go to Darren Ireland for the language revision of the English manuscript.

\section{References}

[1] Somerville, A.L., Wright, D.H., Rotschafer, J.C., 1999. Implications of vancomycin degradation products on therapeutic drug monitoring in patiens with end-stage renal disease. Pharmacotherapy 19, 702-707.

[2] Bucholtz, H.W., Engelbrecht, H., 1970. Über die Depotwirkung einiger Antibiotika bei Vermischung mi dem Kusthartz Palacos. Chirurg. 41, 510-515.

[3] van de Belt, H., Neut, D., Schenk, W., van Horn, J.R., van der Mei, H.C., Busscher, H.J., 2000. Gentamicin release from polymethylmethacrylate bone cements and Staphylococcus aureus biofilm formation. Acta Orthop. Scand. 71, 625-629.

[4] Moojen, D.J.F., Hentenaar, B., Vogely, H.C., Verbout, A.J., Castelein, R.M., Dhert, W.J.A., 2008. In vitro release of antibiotics from commercial PMMA beads and articulating hip spacers. J. Arthroplasty 23 (8), 1152-1156.

[5] Shi, M., Kretlow, J.D., Nguyen, A., Young, S., Baggett, L.S., Wong, M.E., Kasper, F.K., Mikos, A.G., 2010. Antibiotic-releasing porous polymethylmethacrylate constructs for osseous space maintenance and infection control. Biomaterials 31, 4146-4156.

[6] Chang, C.C., Merritt, K., 1992. Microbial adherence on poly(methyl methacrylate) (PMMA) surfaces. J. Biomed. Mater. Res. 26, 197-207.

[7] Oga, M., Arizono, T., Sugioka, Y., Naylor, P.T., Myrvik, Q.N., Gristina, A.G., 1992. The inhibition of bacterial adhesion to a tobramycin-impregnated polymethylmethacrylate substratum. J. Long Term Eff. Med. Implants, 1, 321-328.

[8] Harris, L.G., Richards, R.G., 2006. Staphylococci and implant surfaces: a review. Injury 37, 3-14.

[9] Spicer, P.P., Shah, S.R., Henslee, A.M., Watson, B.M., Kinard, L.A., Kretlow, J.D., Bevil, K., Kattchee, L., Bennett, G.N. Demian, N., Mende, K., Murray, C.K., Jansen, J.A., Wong, M.E., Mikos, A.G., Kasper, F.K., 2013. Evaluation of antibiotic releasing porous polymethylmethacrylate space maintainers in an infected composite tissue defect model. Acta Biomater. 9, 8832-8839.

[10] Kim, S.B., Kim, Y.J., Yoon, T.L., Park, S.A., Cho, I.H., Kim, E.J., Kim, I.A., Shin, J.W., 2004. The characteristics of a hydroxyapatite-chitosan-PMMA bone cement. Biomaterials 25, 5715-5723.

[11] Chan, B.P., So, K.F., 2005. Photochemical crosslinking improves the physicochemical properties of collagen scaffolds. J. Biomed. Mater. Res. 75A (3), 689 - 701.

[12] McDade, J.K., Brennan-Pierce, E.P., Ariganello, M.B., Labow, R.S., Lee J.M., 2013. Interactions of U937 macrophage-like cells with decellularized pericardial matrix materials: Influence of crosslinking treatment. Acta Biomater. 9, 7191-7199.

[13] Yoo, J.S., Kim, Y.J., Kim, S.H., Choi, S.H., 2011. Study on genipin: A new alternative natural crosslinking agent for fixing heterograft tissue. Korean J. Thorac. Cardiovasc. Surg. 44, 197-207.

[14] Kew, S.J., Gwynne, J.H., Enea, D., Abu-Rub, M., Pandit, A., Zeugolis, D., Brooks, R.A., Rushton, N., Best, S.M., Cameron, R.E., 2011. Regeneration and repair of tendon and ligament tissue using collagen fibre biomaterials. Acta Biomater. 7, 3237-3247.

[15] Koob, T.J., Hernandez, D.J., 2002. Material properties of polymerized NDGA-collagen composite fibers: development of biologically based tendon constructs. Biomaterials 23, 203-212. 
Suchý T., Šupová M., Klapková E., Horný L., Rýglová Š., Žaloudková M., Braun M., Sucharda Z., Ballay R., Veselý J., Chlup H., Denk F. (2Ol5) The sustainable release of vancomycin and its degradation products from nanostructured collagen/hydroxyapatit composite layers. J Pharm Sci, in press. DOI: 10.1016/SOO22-3549(15)00175-6, Publisher link: http://dx.doi.org/10.1016/S0022-3549(15)00175-6

Accepted manuscript version.

[16] Backes, D.W., Aboleneen, H.I., Simpson, J.A., 1998. Quantitation of vancomycin and its crystalline degradation products (CDP-1) in human serum by high performance liquid chromatography. J. Pharm. Biomed. Analysis 16 (8), 1281-1288.

[17] Stigter, M., Bezemer, J., de Groot, K., Layrolle P., 2004. Incorporation of different antibiotics into carbonated hydroxyapatite coatings on titanium implants, release and antibiotic efficacy. J. Control. Rel. 99, 127-137.

[18] Chai, F., Hornez, J.C., Blanchemain, N., Neut, C., Descamps, M., Hildebrand, H.F., 2007. Antibacterial activation of hydroxyapatite (HA) with controlled porosity by different antibiotics. Biomol. Engin. 24, 510-514.

[19] Lian, X., Liu, H., Wang, X., Xu, S., Cui, F., Bai, X., 2013. Antibacterial and biocompatible properties of vancomycin-loaded nano-hydroxyapatite/collagen/poly (lactic acid) bone substitute. Prog. Natur. Sci. Mater. Int. $23(6), 549-556$.

[20] Pon-On, W., Charoenphandhu, N., Teerapornpuntakit, J., Thongbunchoo, J., Krishnamra, N., Tang, I.M., 2013. In vitro study of vancomycin release and osteoblast-like cell growth on structured calcium phosphate-collagen. Mater. Sci. Eng. C 33, 1423-1431.

[21] Chen, D.W., Hsu, Y.H., Liao, J.Y., Liu, S.J., Chen, J.K., Ueng, S.W.N., 2012. Sustainable release of vancomycin, gentamicin and lidocaine from novel electrospun sandwich-structured PLGA/collagen nanofibrous membranes, Int. J. Pharm. 430, 335-341.

[22] Melichercik, P., Klapkova, E., Landor, I., Judl, T., Sibek, M., Jahoda, D., 2014. The effect of vancomycin degradation products in the topical treatment of osteomyelitis. Bratisl. Lek. Listy 115 (12), $796-799$.

[23] Lee, J.M., Boughner, D.R., Courtman, D.W., 1984. The glutaraldehyde-stabilized porcine aortic valve xenograft: II. Effect of fixation with or without pressure on the tensile viscoelastic properties of the leaflet material. J. Biomed. Mater. Res. 18, 79-98.

[24] Olde Damink, L.H.H., Dijkstra, P.J., van Luyn, M.J.A., van Wachem, P.B., Nieuwehuis, P., Feijen, J., 1996. Crosslinking of dermal sheep collagen using a water-soluble carbodiimide. Biomaterials 17, 765-773.

[25] Koob, T.J., Willis, T.A., Hernandez, D.J., 2001. Biocompatibility of NDGA-polymerized collagen fibers. I. Evaluation of cytotoxicity with tendon fibroblasts in vitro. J. Biomed. Mater. Res. 56, 31-39.

[26] Sahu, S.C., Ruggles, D.I., O’Donnell, M.W., 2006. Prooxidant activity and toxicity of nordihydroguaiaretic acid in clone-9 rat hepatocyte cultures. Food Chem. Toxicol. 44, 1751-1757. 